\title{
GOBERNANZA, CIENCIA, TECNOLOGÍA Y POLÍTICA: TRAYECTORIA Y EVOLUCIÓN
}

\author{
Emilio Muñoz \\ Departamento de Ciencia, Tecnología y Sociedad \\ Instituto de Filosofia, CSIC
}

ABSTRACT The concept of governance has gained momentum in the political arena, both in the academic realm and in the daily management. The concept sees its origin in the North-American political science school and public administration. Europe has imported the concept as a means to develop new strategies for the management of the relationships between public and private affairs as well as between government and society. The concept is complex both in its foundations and instruments and very likely aims to bridge the social protection system with the strong liberalism.

The introduction of the concept within the science and technology domain has been pushed by the European dynamics. The European policies have driven its integration into the social discourse, essentially through a follower attitude, lacking any analytical reflection and deep analysis of its meaning and consequences.

In this context, the concept seems to be tightly linked to a systemic and complex vision of the management of science and technology that has to be confronted to the existence of a series of levels. It seems pertinent to delve into the relationship of the concept of governance with other fundamental aspects of the scientific and technological issues as being demanded by the principles inspired in the ureflexive modernizationn idea: risk, precaution, uncertainty, sustainability, innovation linked to the intervention of users and consumers.

KEY WORDS Public system, strategic management, techniques, actors, expert community.
RESUMEN El concepto de gobernanza ha irrumpido con fuerza en la arena política, tanto en el ámbito académico como en el de la gestión diaria. El concepto tiene sus raíces en la ciencia política y de la administración norteamericana, y ha sido importado por Europa al reconocer la necesidad de adoptar nuevas estrategias para la administración de las relaciones entre lo público y lo privado, entre el gobierno y la sociedad. El concepto es complejo tanto en sus fundamentos como en sus instrumentos, y probablemente persigue el servir de puente entre el sistema de protección social y el de pleno liberalismo.

La irrupción en el ámbito de la ciencia y la tecnología ha sido promovida por la dinámica política de la Unión Europea. La influencia de la política comunitaria ha facilitado la incorporación del término de modo seguidista, con escasos aportes analíticos y explicativos.

En esta aproximación, el concepto parece estar estrechamente relacionado con la visión sistémica y la complejidad de una gestión de la ciencia y la tecnologia que debe afrontar diferentes niveles. Es preciso, sin embargo, profundizar en las relaciones entre el concepto de gobernanza y otros conceptos cada vez más esenciales para la gestión democrática de la ciencia y la tecnología, que reclama una sociedad que se mueve de acuerdo con principios relacionados con la «modernización reflexivan: riesgo, precaución, incertidumbre, sostenibilidad, innovación asociada a la intervención de usuarios y consumidores.

PALABRAS CLAVE Sistema público, gestión estratégica, técnicas, actores, comunidad experta.

\section{Introducción}

La importante y rápida irrupción -reaparición quizá seria más correcto decir, aunque para confirmar este extremo se requeriría una investigación histórica- del término "gobernanza" en la arena política europea exige una reflexión acerca de su significado y de las razones que están detrás de este proceso.

Simples consideraciones semánticas ponen de manifiesto la conexión del término "gobernanza" con el de "gobierno" con el que los paises con idiomas de origen latino estamos más familiarizados. De hecho, la palabra "gobernanza» ha estado históricamente incorporada en el Diccionario de la Academia de la Lengua Española como una segunda acepción de "gobierno", aunque su uso ha sido muy reducido, como lo prueba el hecho de que no haya sido recogida en el Diccionario del Español Actual (Seco y cols., 1999); no ha formado parte del lenguaje coloquial de los políticos con lo que consecuentemente tampoco ha encontrado un reflejo en los medios de comunicación. Por otra parte, no he encontrado un término parecido en la lengua francesa, aunque esta búsqueda ha estado limitada a diccionarios convencionales, es decir, aquellos que reflejan el uso del lenguaje cotidiano. 
El origen del concepto está probablemente en el ámbito anglosajón. Una consulta a diccionarios convencionales británicos (Websters Third International Dictionary, 1986) nos define "governance" como "the act or process of governing" ("acto o proceso de gobernarn) en su primera acepción, es decir una definición autocontenida, que se extiende por otras tres acepciones que van en el mismo sentido pero trasladando el concepto a nivel de persona o autoridad, a la situación (estar gobernado), a la manera o método de gobernar (expresión personal). Es en la quinta acepción, que se refiere a "system of governing" ("sistema de gobernarn), donde se encuentran probablemente las raices de la relevancia y actualidad del concepto, tal como se ha promovido desde la actividad científica y académica de la corriente norteamericana de la ciencia política y de la administración, como trataremos de desarrollar más adelante ${ }^{1}$.

Sin embargo, la edición del año 2001 del Diccionario de la Lengua de la Real Academia (Real Academia Española, 2001) lo recoge como primera acepción con una definición que se aproxima a la visión dinámica del término governance en la línea anglosajona - sistema de gobernar; aparece registrada en dicho Diccionario como «arte o manera de gobernar que se propone como objetivo el logro de un desarrollo económico, social e institucional duradero, promoviendo un sano equilibrio entre el estado, la sociedad civil y el mercado de la economían.

Desde el punto de vista contextual, la definición de la Academia Española parece tener una estrecha relación con cuestiones y logros políticos propios de la época anterior a la actual -de pleno liberalismo- en que prevaleció el concepto de "estado del bienestar», bajo el impulso de los programas de gobierno de corte socialdemócrata, como herencia de la concepción programática del "Estado providencia", el sistema complejo de la "protección social»" de que se dotaron las sociedades avanzadas a lo largo del siglo XIX, como fruto de una nueva racionalidad jurídica y política.

Parece que la emergencia en el área europea del concepto de "gobernanza" tiene que ver con la necesidad de conciliar los cambios en la situación sociopolítica y económi$\mathrm{ca}$, que se caracteriza por el predominio de la globalización, y la creciente influencia de las doctrinas y políticas ultraliberales, etiquetadas como "neoconserva- durismon, con la fuerte reacción pública, aunque tenga todavía un carácter parcial, que reclama una mayor intervención y participación social.

En lo que sigue, trataremos de trazar el concepto de gobernanza, sus raices científicas, geográficas e históricas, además de plantear las posibilidades técnicas que ofrece su aplicación.

\section{La senda del concepto "gobernanza"}

El concepto encuentra su origen en la tradición de la escuela norteamericana sobre estudios de administración pública. A partir del análisis de algunas obras básicas (Moore, 1995) se observa la utilización habitual del término «democratic governance" ("gobernanza democrática») en los trabajos sobre "gestión estratégica del gobierno", lo que implica en esencia una revisión profunda del papel de lo público en conexión con la consecución de valor y la consiguiente reformulación de la forma de gestionar lo público con el planteamiento de las tareas de los gestores públicos para acomodarse a esa nueva visión.

Partiendo de la constante referencia que lo privado representa en la sociedad norteamericana, los politólogos norteamericanos de esta escuela (por ejemplo, la J.F. Kennedy School of Government de la Universidad de Harvard) han buscado la analogía de la gestión privada con la gestión de lo público con la idea básica de que la administración pública genera valor, e incide, por lo tanto, sobre clientes y consumidores, por lo que debe gestionarse con pautas similares o relacionadas a las que operan en el mundo privado. En contra de la tendencia europea a contraponer sociedad civil y poder público, esta escuela nórteamericana propone para la "gestión estratégica de gobierno" la colaboración entre ambos sectores.

El recorrido sobre la literatura procedente de esta forma de pensamiento ha permitido elaborar el Cuadro adjunto (cuadro $n^{\circ} 1$ ) que cumple, en nuestra opinión, al menos dos objetivos: a) sentar las bases para desarrollar y valorar el análisis de procesos y casos de gobernanza en general, y de modo particular en el ámbito de la ciencia y la tecnología; b) desgranar los argumentos que caracterizan a la gobernanza en el gobierno democrático de lo público. 
CUADRo $n^{\circ}$ 1. La "gobernanza» como forma de gestión de lo público. Factores que contribuyen a definir y producir valor público (los bienes colectivos)

\begin{tabular}{|c|c|c|}
\hline Conceptos & $\begin{array}{c}\text { Misiones } \\
\text { (objetivos) }\end{array}$ & Técnicas / Actores \\
\hline $\begin{array}{l}\text { 1. Capacidad de gestión y } \\
\text { liderazgo en el sistema público. }\end{array}$ & $\begin{array}{l}\text { a) Estrategia } \\
\text { - Legitimación } \\
\text { - Decisiones politicas } \\
\text { sustantivas } \\
\text { - Construcción de } \\
\text { capacidades } \\
\text { operativas } \\
\text { b) Declaración de misiones } \\
\text { c) Criterios de evaluación }\end{array}$ & $\begin{array}{l}\text { - Mantener enfoque } \\
\text { gestionario } \\
\text { - Identificar tareas claves } \\
\text { de carácter administrativo } \\
\text { y politico } \\
\text { - Movilizar apoyo externo e } \\
\text { interno } \\
\text { - Niveles de abstracción en } \\
\text { definición } \\
\text { - Grado de riesgo y } \\
\text { exposición } \\
\text { - Visión y propósito }\end{array}$ \\
\hline $\begin{array}{l}\text { 2. Construcción de apoyo y } \\
\text { legitimidad }\end{array}$ & 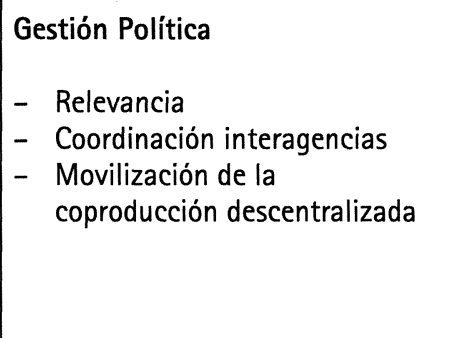 & $\begin{array}{l}\text { - Procesos toma de } \\
\text { decisiones } \\
\text { - Aprovechamiento de ideas } \\
\text { generales y saber } \\
\text { convencional } \\
\text { Supervisores } \\
\text { Grupos de interés }\end{array}$ \\
\hline 3. Gestión política y su entorno & $\begin{array}{l}\text { Ética y desarrollo de la acción } \\
\text { política }\end{array}$ & $\begin{array}{l}\text { - Búsqueda de apoyo } \\
\text { - Gestión del desarrollo de } \\
\text { políticas } \\
\text { - Negociación } \\
\text { - Deliberación pública, } \\
\text { aprendizaje social, } \\
\text { liderazgo } \\
\text { - Mercado y sector público, } \\
\text { comunicación estratégica }\end{array}$ \\
\hline
\end{tabular}

Fuente: Elaboración propia a partir de M. H. Moore (1995)

\section{Bases del concepto de gestión de lo público en relación con la producción de bienes}

La escuela norteamericana de estudios sobre administración pública ha puesto el énfasis en la mejora del rendimiento de lo público («management performance»). Este rendimiento de la gestión es considerado como la variable dependiente, mientras que a las estructuras institucionales se les atribuye el carácter de variable independiente. De acuerdo con estas claves, las opciones ante la ejecución de las obligaciones a que se enfrenta lo público contraponen la ureforma institucional" a la "gestión mejorada». 
Las aproximaciones analíticas se abordan desde una perspectiva multidisciplinar en la que coexisten diversas disciplinas y líneas. Desde el prisma de la ciencia politica se exploran las caracteristicas de los ambientes en que operan los gestores: contexto político, instituciones legislativas, prensa y motivaciones. La literatura económica favorece en el plano teórico el examen de las relaciones entre gobierno y sociedad y la profundización en los métodos para evaluar; entre los que cabe mencionar los incentivos para gestores y las negociaciones. En el marco de la teoria de la organización se contempla el análisis del comportamiento de lo público y lo privado, mientras que el área de la administración pública analiza la teoría de la gestión pública en democracia (filosofía de la gestión pública) con el objetivo de asegurar el control democrático respecto a la consistencia, eficacia y eficiencia de la administración pública.

Dos corrientes predominan en la forma de entender la administración pública. Una descansa en las organizaciones, la cual al plantearse cambios en el rendimiento organizativo ("performance"l) se enfrenta a la difícil correlación con actividades de gestión en las que intervienen muchos factores y con gestores que son funcionarios, que, por lo tanto, no gozan de la legitimidad de la elección. Ello hace prevalecer la rutina y dificulta la introducción de prácticas innovadoras.

Una segunda se basa en las políticas, en un contexto en el que el poder y la legitimación de la posición política dependen del entorno político. Ello determina la intervención de un mayor número de actores y reclama actuaciones variadas en función de coyunturas distintas.

La gestión de lo privado se enfrenta continuamente a esta situación en la que la organización tiene que afrontar coyunturas cambiantes, lo que determina la necesidad de estrategias (políticas). De ahi que la gestión estratégica del "sector público" en la línea de la escuela que estamos analizando, busque la inspiración en lo privado y reclame posiciones en los gestores ${ }^{3}$ que guarden relación con las expectativas que la sociedad ve en los gestores privados: producen bienes con un valor que tiene que ser reconocido por la sociedad, los consumidores.

Bajo este prisma, los gestores de lo público deben de cambiar para ser importantes actores innovadores con la asunción de la responsabilidad de los cambios que tienen que llevar a cabo las organizaciones públicas, y de cómo esos cambios deben ponerse en práctica en lugar de ser garantes inerciales de las organizaciones. Los gestores públicos pasan de ser técnicos a se estrategas.

La aproximación alternativa del gobierno y función de la administración pública descansa en buscar, explorar y explorar oportunidades de "crear valor público", es decir, de ejercer un gobierno movido (dirigido) por los usuarios (los consumidores).

\section{Definición de valor público}

Lo que antecede es más fácil de decir que de hacer. La dificultad se acrecienta cuando vivimos en una sociedad que aplaude el consumo privado con mayor fuerza que la consecución de objetivos comunes, que iddolatra la libertad individual y atribuye a la empresa privada un papel motor del desarrollo económico y social superior al del gobierno. Por ello, la gestión de lo público y la provisión de recursos para ello se hacen con tacañeria. Consecuentemente, los gestores (actores) públicos deben ofrecer resultados que no sólo son valorados, sino que deben poner de relieve que los resultados obtenidos satisfacen el coste que puedan representar los costes para el consumo privado o para una inevitable restricción de libertad. Este es el único modo de estar seguros de que se ha producido «valor público».

Bajo estas coordenadas, el objetivo de lo que hay que llamar "gobierno estratégico" es la creación de valor público y de valor social, con repercusión en bienes colectivos, como son defensa, seguridad, limpieza, educación, medio ambiente, salud, protección de los desastres. Si se me permite un inciso, dentro de esta reflexión general, sobre el tema de la ciencia y la tecnologia cabe decir que el desarrollo científico y técnico tiene una creciente relación con los bienes colectivos (Muñoz, 2002) y con ello se interrelaciona y hace dependiente de otras políticas: económica, sanitaria, medioambiental, de seguridad.

\section{El «mercado» político: ciudadanía y consumo colectivo}

El siguiente paso es identificar y determinar quienes son los clientes a los que ofrecer los bienes públicos. A diferencia de lo que ocurre en el sector privado donde son los individuos quienes deciden que un producto merece la pena e invierten en su compra, pagando un precio que compensa al productor (la empresa) de los costes en los que incurre,

ARBOR CLXXXI 715 SEPTIEMBRE-OCTUBRE (2005) 287-300 ISSN: 0210-1963 
en el sector público, los individuos no escogen ni contribuyen voluntariamente como consumidores a la producción de los bienes públicos. En este caso, los recursos llegan por la vía coercitiva de los impuestos, un hecho que crea problemas para valorar las actividades de gobierno. La imposibilidad, de hecho la renuncia, de que los recursos públicos procedan de la "voluntad" (elección) individual, determina que sean las instituciones y los procesos de la democracia representativa los que hagan factible la conjunción de los individuos y la decisión colectiva sobre lo que quieren alcanzar juntos sin sacrificar sus aspiraciones individuales.

La conciliación entre las tensiones que existen entre el deseo de tener políticas democráticas y los riesgos de que estas políticas sean vulnerables a diferentes tipos de corrupción es un desafío permanente que exige la aplicación de diferentes criterios para estimar la adecuación de la gestión para el valor público.

Entre ellos cabe mencionar siguiendo una lógica histórica:

i) Conseguir los objetivos fijados de modo normativo de manera eficiente y efectiva.

ii) La separación entre política y administración como esferas independientes que deben perfeccionar su actividad de acuerdo con pautas propias.

iii) La desconfianza generada por y ante la política ha supuesto la emergencia de una nueva plataforma para la racionalización de la política democrática. Esta plataforma se ha establecido sobre una nueva clase de conocimiento experto. La nueva fórmula se apoya en un conjunto de técnicas analiticas procedentes de distintas áreas disciplinares: economia, estadistica, investigación operativa. Las nuevas técnicas abarcan el análisis político, la evaluación de programas, el análisis coste/eficacia y el análisis coste/beneficio.

iv) Maximizando las analogías con el sector privado, los administradores públicos han abrazado los objetivos de servicio al consumidor y se han comprometido a determinar el valor de sus esfuerzos para satisfacer a sus "clientes".

v) La justificación de un criterio gestor para el valor público descansa en seis puntos:

- El valor tiene sus raices en la percepción y deseos de los individuos.

Hay diferentes tipos de deseos que satisfacer. Las aspiraciones colectivas se canalizan por las organizaciones públicas y se despliegan por la autoridad pública que obliga a los ciudadanos o utiliza los recursos de los impuestos para objetivos que han sido aprobados por los ciudadanos y gobiernos representativos. Por otro lado, las aspiraciones individuales se vehiculan a través del mercado.

- La gestión del sector público puede crear valor, incluso por la producción de cosas que benefician a clientes especificos.

- La producción y distribución debe responder a criterios de juego limpio y eficacia.

- La producción de valor (bienes públicos) está íntimamente asociada a la política. La política es la respuesta que una sociedad democrática liberal ha formulado a la pregunta (analíticamente cuasi insoluble) de qué cosas deben producirse para propósitos colectivos con recursos públicos.

- El ámbito operacional del sector público experimentará cambios. Las aspiraciones de los ciudadanos experimentarán modificaciones como ocurrirá con los métodos para afrontar viejas tareas. Como los gestores del sector privado, los gestores en el sector público deben trabajar arduamente en la definición de iniciativas valiosas según parámetros públicos, así como en la producción de esos valores. Deben además estar preparados para adaptar y recolocar sus organizaciones en sus entornos políticos y de trabajo además de asegurar su continuidad.

En resumen, los objetivos básicos de esta orientación gestora de lo público son: hacer visible el valor de lo público con una serie de técnicas específicas, movilizar y aprender de la política y reestructurar las organizaciones.

\section{Definición de la misión y de los objetivos del sector público}

La estrategia organizativa del sector público responde a la necesidad que tienen los gestores de dar cuenta del valor de lo que sus organizaciones producen. Esta necesidad tropieza con la dificultad de que no pueden estar seguros de lo que ese valor es (Dahl, 1947; Wilson, 1989) ${ }^{4}$. Ello deriva en una ambigüedad fundamental, tanto en lo que concierne a los fines como a los medios. Esta ambigüedad es fuente de una cierta discrecionalidad y abre asi una oportunidad para el liderazgo.

El sector privado afronta idéntica problemática. La sociedad suele aceptar más fácilmente el liderazgo en el sector pri- 
vado que en el público. Los ejecutivos responden a esta situación con el establecimiento de objetivos estratégicos y con el desarrollo de planes operativos para sus organizaciones. Tres conceptos fundamentales: estrategia corporativa (posicionamiento), estrategia en complejos o conglomerados diversificados, y estrategia como reflejo de un acuerdo sostenible entre una variedad de actores, configuran esta dinámica.

En este sentido, la Kennedy School of Government (Harvard) ha desarrollado un concepto rudimentario de estrategia organizativa para el sector público (Moore, 1995) ${ }^{5}$.

Esta estrategia se apoya en la asunción, dentro de la acción del gestor, del llamado triángulo estratégico. De acuerdo con esta propuesta, la estrategia debe ser valorable en términos sustantivos - produce cosas con buena relación coste/beneficio-; legítima y sostenible políticamente -atrae de modo continuo recursos y autoridad por parte del entorno político al que se debe-; factible en términos operativos y administrativos -se puede llevar a cabo con la organización disponible y una ayuda externa proporcionada.

\section{Del gobierno estratégico a la "gobernanza»}

Esta trayectoria demanda la construcción de apoyo y legitimidad. Para ello se hace preciso una incursión en el dominio de la política, entendiendo este término no sólo como la proyección de las esperanzas y deseos de los ciudadanos y de sus representantes, sino que debe incluir todos los acuerdos políticos que están incorporados en la legislación que enmarca y define la acción de los gestores políticos. La importancia de las funciones de la gestión política descansa en los siguientes argumentos.

- Los gestores públicos deben frecuentemente incorporar actores que están fuera de su ámbito de autoridad con el fin de alcanzar sus objetivos, por ejemplo, solicitar permiso para utilizar recursos, ayuda operativa para alcanzar los resultados.

- Las instituciones políticas son las que atribuyen los recursos para la realización de programas. Son necesarios, por ejemplo, canales para "rendir cuentas» o para autorizar cambios e innovaciones.

- Los gestores se deben a la política y a la ley. En este sentido, deben actuar con y promover la coordinación entre agencias, así como procurar la movilización de la coproducción descentralizada.
Este elemento es particularmente relevante en aquellos casos y temas en que la capacidad de actuación está profusamente descentralizada. En este caso, los gestores no pueden apalancar la capacidad operativa que necesitan a través de la persuasión del superior que debe dictar las órdenes, sino que necesitan llegar a cientos, miles, quizá millones de interlocutores implicados en la toma de decisiones. Este tipo de gestión descentralizada, cada vez más relevante en el contexto político de una sociedad globalizada, difiere notablemente de la visión de la gestión política asociada con los conceptos burocráticos de defensa y apoyo, y capacidad empresarial

\section{Actores en la gestión política}

Los actores relacionados con la gestión política varian considerablemente dependiendo de los temas, los propósitos y los tiempos.

En el centro de la gestión política están las autoridades que nombran a los gestores, establecen los términos de referencia para la responsabilidad de su gestión y proveen los recursos necesarios. Parlamentarios, ministros, consejeros son las figuras más relevantes en este epígrafe.

En otro plano más alejado pero quizá más arriesgado en términos relacionales, figuran los medios de comunicación. La acción de los medios fuerza a los gobiernos a ser más abiertos, más transparentes, en lo que concierne a las acciones políticas y a la determinación de los valores que encierran, por lo que serian un factor importante en la configuración de la "gobernanza democrática". La prensa refuerza más bien que debilita la acción política gubernamental.

La evidencia de que se dispone para cerrar este debate es poco clara6: hay casos que revelan el impacto de la prensa en los procesos de toma de decisiones. En general, las influencias, si existen, se manifiestan en el "espacio temporal" y en el "proceso" de la toma de decisiones, más que en los resultados finales.

Las asociaciones de ciudadanos y los grupos de interés forman parte del entorno de autoridad (Wilson, 1973; Truman, $1951)^{7}$. El debate sobre el papel de estos grupos de interés en la "gobernanza democrática" está de plena actualidad. Las primeras criticas se centraron en el poder de los intereses económicos y ciertas empresas en la política guberna- 
mental. Estas criticas se han ido atenuando con la introducción de leyes en el ámbito laboral, el establecimiento de agencias reguladoras y la emergencia de grupos preocupados por cuestiones de ámbito público como el medio ambiente, los consumidores, la limitación fiscal, la igualdad de las mujeres, o la defensa de los jóvenes. De modo inesperado, las preocupaciones generales de los ciudadanos han encontrado vehículos organizados para su expresión (Olson, $1965)^{8}$.

\section{Combinación de valores e intereses diversos}

Los intereses, las peticiones y expectativas de quienes integran los entornos de autoridad y de coproducción constituyen los materiales que los gestores/actores políticos deben usar para configurar mandatos poderosos y efectivos. Es relevante, a este respecto, el modo en que los intereses se combinan a través de procesos formales de toma de decisiones, o por medio de acuerdos establecidos, procedentes del acervo del buen juicio sobre la mejor práctica para afrontar ciertos problemas.

\section{i) Proceso de toma de decisiones}

La legitimación en el proceso supone un mayor apoyo para la decisión, que será de este modo más difícil de ignorar o revertir $y$, consecuentemente, el mandato será más fuerte.

La legitimidad formal, la eficiencia histórica y la intervención de participantes poderosos tienden a ser los soportes de la legitimación de un proceso de toma de decisiones. Pero esta legitimación y la fuerza de un proceso de toma de decisiones también puede ser influida por el grado de incorporación de cualidades abstractas, que el público asocia con la naturaleza apropiada de un proceso de toma de decisiones. Estas expectativas del público se relacionan con una serie de valores de compromiso público como: representación política; uso eficiente de experiencia relevante; respeto por los antecedentes; determinación y claridad en las decisiones; transparencia y apertura en las deliberaciones; sensibilidad ante la experiencia acumulada, etc.

ii) Ideas públicas y sabiduría convencional

Los intereses de la gran diversidad de actores que intervienen en el entorno de autoridad se pueden combinar para dar lugar a un mandato por medio de la existencia de niveles comunes de entendimiento respecto a la naturaleza de un problema y la mejor forma de tratarlo. El conocimiento convencional (el juicio convencional) juega un papel importante en la consecución de la legitimidad de una opción política $o$ en determinar su arrinconamiento.

De modo análogo a lo que ocurre con los grupos de interés, los gestores pueden enfrentarse a las ideas públicas 0 , por el contrario, utilizarlas. Si la política propuesta por un gestor es consistente con lo que se acepta como verdad, la política gozará de más legitimidad que si se opone al acervo común. Si, por el contrario, la política va en contra del sentido común, el gestor deberá encontrar vías para contrarrestar la fuerza del sentido común. Esta situación no es sencilla ni la solución es rápida.

\section{La dinámica del entorno de autoridad}

En un momento dado, el entorno de autoridad, que guía y apoya a los gestores, tiene una composición y una configuración determinadas. Los mandatos cambian. Las coaliciones políticas que apoyaron una politica o una estrategia organizativa pueden erosionarse como consecuencia de las fluctuaciones del poder político y de las evoluciones en las posiciones de los actores políticos. Grupos cuyos intereses carecieron de aceptación en un principio pueden evolucionar, ganando poder. Pueden surgir nuevos grupos que defiendan intereses que van en la trayectoria del gobierno. Del mismo modo, se acumula experiencia en relación con políticas particulares y estrategias organizativas, experiencia que empieza a ejercer influencias sobre su adecuación y oportunidad. El cambio en el acervo de conocimiento sobre politicas buenas o eficientes determina cambios en los mandatos.

\section{El desafío de la gestión política}

El espacio en el que se gestiona estratégicamente la politica no es un espacio cómodo ni seguro. Se corresponde con un mundo complejo en el que coexisten varios niveles y una diversidad de actores. Los gestores políticos deben interaccionar, aunque sea parcialmente, con este mundo. Esta interacción será tanto mayor cuanto más grande sea el esfuerzo para acomodarse a la definición de gobernanza, la cual por esencia reclama una amplia participación de actores si se quiere un desarrollo que combine las iniciativas e intereses de la sociedad, el Estado y el mercado. 
Para avanzar por este camino, los responsables políticos deben poner en acción, movilizar a los ambientes externos a la acción política directa, ¿pero cómo conseguirlo? es la cuestión fundamental, una pregunta que tiene una respuesta técnica pero también ética.

Las técnicas deben servir para poner de manifiesto que los actores políticos tratan de alcanzar objetivos públicos, y no sus propios intereses o su propia visión de cuáles son los valores públicos que están en litigio.

A continuación, desgranaremos las principales técnicas que permiten conseguir esta gestión política estratégica, presentación en la que ofreceremos, de modo breve, las características y la táctica de cada instrumento, a la par que se exponen algunas críticas a cada uno.

\section{Búsqueda de apoyo}

Con esta técnica (en inglés "entrepreneurial advocacy"), los responsables políticos actúan según el plano de la menor generosidad. Ofrecen sus propuestas y buscan para sus políticas preferidas el apoyo de las autoridades y representantes significativos para conseguir la adopción de tales políticas con un apoyo sólido y basado en la autoridad.

La crítica fundamental al concepto de "entrepreneurial advocacyn concierne a la desviación de los objetivos que puede tener lugar a causa de la influencia de los que apoyan la propuesta, que pueden llegar a manipular las decisiones gubernamentales para ajustarlas a sus deseos.

Se puede concluir que la técnica de "búsqueda de apoyo" da una buena pista para analizar y diagnosticar los escenarios políticos, aunque las tácticas para su puesta en práctica carecen de la fuerza moral que se espera de una buena gestión democrática.

\section{Desarrollo de una política de la gestión}

Ante estas críticas, el desarrollo politico de la gestión aflora como aproximación alternativa. La diferencia fundamental, con respecto al anterior instrumento, radica en que, en esta segunda técnica, los actores políticos no tienen una opción preconcebida, procuran, por lo tanto, gestionar un proceso que confiera legitimidad-combinación de poder más adecuación- a una decisión.

ARBOR CLXXXI 715 SEPTIEMBRE-OCTUBRE (2005) 287-300 ISSN: 0210-1963
Las tácticas para avanzar en esta dirección son, $\sin$ duda, más complejas que en el caso anterior. Es preciso poner en práctica un amplio proceso de consultas; conseguir superar las dificultades inherentes a ese proceso con la consecución de una posición, de forma que el proceso de consultas conduzca a un cierre; el establecimiento de comisiones como mecanismo para esos fines; poner de relieve la importancia del proceso analítico; y hacer frente a la incertidumbre.

Las críticas principales a esta alternativa de desarrollo político surgen alrededor del excesivo formalismo y mecanización que llevan consigo. Una de las asunciones presentes en esta aproximación es que los intereses se van a ir construyendo hasta alcanzar el acuerdo sobre puntos básicos. Los críticos a esta aproximación política a la gestión contraponen el paradigma de la negociación multilateral.

\section{Negociación}

Esta táctica ha permanecido ignorada como instrumento político por tres razones principales. En primer lugar, los analistas asumieron, de modo implícito o explícito, que los individuos negociaban por su propio interés. Por ejemplo, la consecución de un precio determinado por un bien personal. En segundo lugar, los analistas estuvieron más preocupados por la predicción y evaluación de los resultados de la negociación que por la propuesta de elementos tácticos para los negociadores. Tercero, los analistas focalizaron el análisis sobre situaciones simples, en las que las pretensiones de las partes eran fácilmente identificables. La tendencia actual supone un giro a estos planteamientos, ya que el nuevo paradigma no sólo admite la existencia de una variedad de intereses, sino que anima a que esta variedad de intereses se incorpore a la negociación como reflejo del interés público.

El paradigma de la negociación, no obstante sus indudables valores, también suscita críticas. A pesar de su aparente valor colectivo, las críticas subrayan la tendencia a la preeminencia de visiones individuales a la hora de efectuar la selección. En suma, los críticos proponen que la gente debe ser orientada a que piensen y actúen como "ciudadanos" en lugar de moverse por clientelismos de las decisiones políticas, o por nociones alejadas de lo que es el valor público. 


\section{Debate público, aprendizaje social, liderazgo}

La cuarta aproximación trata de ampliar la base para ir más allá de las posiciones de autoridad. En efecto, en lugar de asumir que los intereses de los particulares y que las posiciones acerca del valor público están definidas, la nueva aproximación asume que estos factores se pueden cambiar por la interacción social.

El avance en esta nueva dirección comporta unas vías de diagnóstico que se resumen a continuación:

- "Debate público», un concepto que apoya la consulta a los ciudadanos.

- Los conceptos de "aprendizaje social" y "liderazgo" guardan una estrecha conexión con las ideas relacionadas con el concepto de "debate o deliberación pública». Todos ellos ponen el énfasis en la intervención de amplios colectivos que se extienden más allá de las estructuras formales de gobierno.

- Los conceptos de "aprendizaje social" y uliderazgo" proponen tanto la movilización de los ciudadanos en su capacidad de acción como en su capacidad organizativa para aconsejar y para alcanzar acuerdos colectivos. En resumen, persiguen la movilización ciudadana para la acción.

La táctica básica para gestionar la adaptación social es la gestión de la trayectoria del aprendizaje. Desde la responsabilidad de gobierno, se debe adecuar la velocidad de la toma de decisiones a la capacidad de aprendizaje de la ciudadanía.

\section{Mercadotecnia en el sector público y comunicación estratégica}

El concepto de aprendizaje social lleva aparejado el establecimiento de un marco en el que los gestores del sector público pueden configurar la gestión política. Este nuevo marco se abarca bajo el titulo «mercadotecnia del sector público" o "comunicación estratégica». El adjetivo estratégica no quiere decir que la comunicación esté diseñada para manipular, sino que se diseña para poner de manifiesto politicas particulares, o estrategias organizativas, con el objetivo de hacer más fácil su comprensión y para aglutinar el apoyo y la cooperación de quienes deben colaborar para conseguir el resultado esperado.
Desde un punto de vista crítico es indudable que existen riesgos al promover que los actores políticos desarrollen una estrategia de comunicación. A partir de ella, se pueden crear falsas expectativas.

Conviene señalar, sin embargo, que existen evidentes ventajas con una estrategia de esta naturaleza, ya que puede aumentar la responsabilidad de los actores ante las expectativas de sus superiores, puede aumentar asimismo el apoyo y el nivel de compromiso para alcanzar los objetivos. El valor de esta estrategia puede contribuir a la promoción de una información seria, veraz, honesta, en lugar de que sea decepcionante, falsa y manipuladora.

\section{8. "Gobernanza", la ruta hacia la definición y producción de valor en lo público}

La gestión política aflora como una función clave en el sector público. Es indispensable para conseguir una acción eficaz en el desempeño de sus funciones en beneficio de la sociedad en sentido general. Pero es también un tema con resonancias éticas. Esta función es indudable porque los actores/gestores políticos deben compartir responsabilidades con un conjunto de actores: otros actores politicos y ciudadanos para decidir lo que valdria la pena producir con recursos públicos y para producir en efecto lo que han declarado como más valioso. Deben interactuar con individuos con los que no tienen relación directa de autoridad para determinar los fines y organizar los medios. La interacción con el público y la sociedad más allá de sus ámbitos directos es fundamental. Al implicarse en la gestión políti$c a$, los gestores deben caminar por una linde que separa dos frentes: por un lado, una escasa capacidad de influencia sobre las autoridades; por otro lado, su excesiva autoridad ante los ciudadanos de modo que ponen en riesgo la calidad de la gobernanza democrática y la libertad del público. Una serie de técnicas: búsqueda de apoyo, gestión de la politica, negociación, debate público, aprendizaje social, y mercadotecnia en el sector público, que se van a mejorar y revisar de modo continuo, ayudan a transitar por esa frontera.

Lo más relevante, en esencia, es tener presente estos conceptos, actividades y técnicas que pueden ser soporte de la respuesta a la pregunta básica sobre cómo los gestores/actores públicos, en un contexto como el actual, actúan para legitimar sus empeños, así como para movilizar a quie- 
nes caen fuera del nivel y rango de autoridad con el fin de alcanzar un éxito lo más amplio posible en la consecución de los objetivos. Estas son las tareas más complicadas y retadoras a las que se enfrentan los actores/gestores públicos.

La creación de valor público ha sido utilizada como argumento para proponer la necesidad de gobierno estratégico en el sector público. Este gobierno estratégico encuentra su proyección integradora en el concepto de gobernanza. De acuerdo con todo lo expuesto, se puede concluir que gobernanza, en una definición ampliada de la que recoge el Diccionario de la Lengua, es la puesta en práctica de formas de gobierno estratégicas para poner de relieve el valor de lo público a través de la relación entre sociedad, mercado y Estado y conseguir de este modo un desarrollo socialmente sostenible. Una serie de técnicas, con un creciente nivel de participación social y que deben estar en proceso de continua revisión y mejora, emergen como puntos de apoyo significativos para la consecución de estos objetivos.

\section{9. "Gobernanza" y ciencia y tecnología}

La implantación del término gobernanza en el ámbito de la ciencia y la tecnología ha llegado de la mano de la dinámica política de la Unión Europea que ha caracterizado los pasos finales del desarrollo del $V$ Programa Marco y el diseño y puesta en marcha del VI Programa Marco. Un conjunto de iniciativas sociopoliticas (comunicaciones, decisiones, identificación y selección de prioridades) promovidas por la Comisión Europea en relación con la reflexión relacionada con la concepción del Espacio Común Europeo en I+D (ERA) han ido configurando un paisaje en el que el concepto gobernanza ha alcanzado carta de naturaleza. A partir de ahí se ha producido una floración de publicaciones en que se relaciona la dinámica de la promoción de la $I+D$, su financiación con la intervención de diferentes formas de agencia y la gestión de los recursos humanos y materiales con dicho concepto de gobernanza. Sin embargo, en esas publicaciones parece que se recurre al uso del término gobernanza de forma cuasi automática, seguidista de las iniciativas promovidas por la Comisión Europea, pero sin aporte analítico ni sustrato descriptivo.

Una de estas publicaciones que responde al sugestivo titulo de "Changing governance of research and technology policy. The European research arean (Edler y cols, 2004) recoge toda una serie de artículos y un número de referencias al término gobernanza. Sólo el artículo introductorio ofrece algunas bases analíticas acerca de lo que representa el concepto de gobernanza como desarrollo de una visión sistémica, derivada de las propuestas de la escuela norteamericana de la ciencia política. Por otro lado, el seguimiento de las referencias a la palabra gobernanza revela que la práctica totalidad de las citas a esa palabra son una simple traslación de las iniciativas europeas, con lo que se carece de elementos explicativos acerca del concepto y de su valor estructural y prospectivo para poder estudiar su influencia en el desarrollo de la ciencia y la tecnología en la sociedad actual. Parece evidente que la misión fundamental que atribuye la propuesta de la Comisión al término gobernanza es su operatividad para afrontar el problema de los diferentes niveles de promoción y gestión de la ciencia y la tecnología, y de su instrumento político -la política científica-tecnológica y la de innovación- en un contexto como el europeo donde coexisten lo supranacional, lo nacional, lo regional e, incluso en ciertos lugares o momentos, lo local. De ahí que la línea elegida por los decisores en política cientifica y tecnológica, y seguida por los estudiosos del tema, sea la que trata de comprender el papel de los "espacios" y la influencia en ellos de la existencia de varios niveles políticos -propuesta, decisión y ejecución- y de la participación de diversos actores. En resumen, el reto de la "coordinación operativa» ante la división del trabajo en «l+D e innovación».

Esta aproximación es interesante, pero parece también, en una primera mirada, limitada en sus fundamentos y en sus aspiraciones. Se presenta asimismo arriesgada, pues responde a un seguidismo mimético por parte de los analistas, con lo que nos podemos ver envueltos en un círculo vicioso: se estudia aquello que los decisores politicos han introducido, mientras que se renuncia a entender lo que sustenta la oportunidad, validez y el potencial del concepto para desarrollar una dinámica racional en términos sociales, económicos y políticos en el ámbito de la ciencia y la tecnología.

No podemos olvidar los contextos sociales de preocupación y contestación en que se mueve la idea de uprogreson y la aplicación de las nuevas tecnologías en las sociedades avanzadas "per se" y en su relación con el mundo en desarrollo, menos avanzado. Temas como riesgo, incertidumbre, responsabilidad, participación social, democratización de la ciencia (¿y la tecnologia?), intervención y "laissez-faire», relación entre lo público y lo privado, patentes y subdesa- 
rrollo, están muy presentes en la conflictiva agenda sociopolítica que marca el periodo comprendido entre el final del siglo XX y los inicios del siglo XXI.

A la vista de lo que se anticipaba acerca de la escasa penetración del concepto gobernanza en la arena política española, los riesgos de seguidismo sin marcos de referencia son aún más evidentes en nuestro país. Por ello, al encargar la Fundación Española para la Ciencia y la Tecnología (FECYT) al Instituto de Filosofía del CSIC (Departamento de Ciencia, Tecnología y Sociedad), un proyecto sobre "gobernanza y sociedad civil en ciencia y tecnología en Españan, la dirección del proyecto estimó pertinente su aceptación por su indudable interés, aunque se planteó, al mismo tiempo, como un primer objetivo revisar y reorientar el proyecto. Como fruto de este primer trabajo, se presentó un Plan de Trabajo en el que se recogen dos elementos estratégicos: a) focalizar el trabajo en ciertos temas como base para profundizar en el valor analítico y prospectivo del concepto gobernanza; b) elaborar estos estudios de caso en marcos teóricos y analiticos que confieran suficiente apoyo para aumentar su trascendencia.

\section{Gobernanza, ciencia y tecnología. Otra mirada}

La definición de "gobernanza" que acabamos de delinear lleva aparejada una clara opción sistémica, aunque esta opción encierra una mayor complejidad que la simple concepción de sistema como conjunto de elementos afines que cooperan. Se concibe como un sistema político, desarrollado por la escuela norteamericana de ciencia politica, en el que se gobierna con la intervención de una variedad de actores y que requiere una arquitectura para la decisión en varios niveles.

En el ámbito de la ciencia y la tecnología, la visión predominante, desde mediados de los años 50 hasta la década de los 70 en esa misma centuria, ha sido el modelo lineal que conectaba ciencia, investigación, desarrollo e innovación en una cadena, en la que cada uno de los eslabones tenía su propia dinámica y actuaba de acuerdo con patrones especíicos, mientras que la influencia sobre el siguiente eslabón se limitaba a un flujo de materiales (conocimiento en términos generales).

Las dificultades experimentadas por el modelo lineal para explicar la influencia de la actividad cientifica-técnica (tec- nocientifica en la nomenclatura de J. Echeverria, 2002, 2003) en la producción tecnológica y en la producción de innovaciones condujo al concepto de usistema de innovación" como modelo interpretativo de tales procesos, promovido por la teoría económica de carácter evolucionista y neoinstitucionalista (Smits y Kuhlmann, 2004).

El concepto de "sistema de innovación» trata de integrar la práctica totalidad de los factores que intervienen en la producción y utilización de ciencia y tecnología para conseguir su transformación en bienestar económico y social. Sin embargo, este concepto, entendido como conjunto de elementos por su naturaleza y estructura, presenta problemas para el caso de la ciencia y la tecnología. En este conjunto existen elementos que pueden y deben cooperar, pero cuya afinidad es cuando menos limitada. De hecho, el concepto está centrado, como su propio nombre indica, en el proceso de innovación y se focaliza en los intereses y estrategias empresariales que en esencia son diferentes de los que movilizan los otros dos elementos del trinomio $I+D+i$, la investigación y el desarrollo tecnológico. En la definición propuesta por Metcalfe (1995) un "sistema de innovación" abarca todas las instituciones implicadas en la investigación cientifica y su desarrollo; instituciones que son responsables de la acumulación y difusión del conocimiento, proceso que encuentra su reflejo en la educación y formación de la ciudadanía con potencial impacto en las condiciones de empleo y trabajo, incide en el desarrollo tecnológico, para por último influir en la producción de nuevos productos y procesos así como en su distribución. Esta concepción de "sistema de innovación" atribuye al conocimiento el papel fundamental, lo considera soporte y armazón para el eficaz funcionamiento de ese sistema.

La atribución de este papel central al conocimiento «experton sirve para poner de manifiesto que no todas las instituciones tienen similar importancia en la configuración y puesta en acción de un "sistema de innovación». La relevancia de la comunidad "tecnocientífica" en la "gobernanzan de la ciencia y la tecnología es evidente ${ }^{9}$, lo que confiere a la "gobernanza" de este ámbito sociopolítico un marcado carácter diferencial con respecto a otros tipos "gobernanza".

Desde hace siglos, los científicos avanzaron en el diseño y puesta en práctica de las instituciones por las que vehicular la difusión de su actividad y establecer plataformas para sus críticas y discusión. El siglo XX ha sido considerado el

ARBOR CLXXXI 715 SEPTIEMBRE-OCTUBRE (2005) 287-300 ISSN: 0210-1963 
siglo de la ciencia en la opinión de relevantes historiadores, véase por ejemplo José Manuel Sánchez Ron (2000). En lo que algunos han llamado "siglo corto" ${ }^{10}$, se ponen en marcha los grandes instrumentos de la política cientifica: la National Science Foundation y los Institutos Nacionales de la Salud en los Estados Unidos, los Consejos sectoriales británicos, la Organización Max Planck en Alemania, el CNRS en Francia, el CSIC, con todas sus limitaciones, en España. A partir de todos estos hechos, los científicos (y tecnólogos) han ido configurándose como actores polivalentes en el sistema de "gobernanza" de la ciencia y la tecnología. Esta polivalencia se manifiesta a través de la ocupación y desarrollo de diferentes puestos y papeles: son usuarios de los planes y programas que financian su actividad, son consumidores de fondos $y$ bienes públicos desde la financiación de sus salarios y de los proyectos hasta el uso de infraestructuras. Los científicos son también, con frecuencia, actores fundamentales en el diseño de políticas asi como en la gestión de las mismas: la gestión de la dinámica de la actividad "tecnocientífica" descansa con frecuencia en miembros de la propia comunidad, la cual se ha dotado de reglas para evaluar las propuestas y resultados de dicha actividad.

Sin embargo, la evolución experimentada por este tipo de procesos de gobierno ha sido influenciada por el cambio en los contextos de desarrollo científico y tecnológico (Muñoz, 2002). La conexión e interacción entre los conceptos de "sistema político" y usistema de innovación" ha determinado la heuristica para analizar el sistema de "gobernanza" en relación con la ciencia y la tecnología. La revisión crítica del modelo lineal ha servido para identificar cuatro brechas 0 desfases entre la linearidad y su repercusión en un entorno complejo. Esas brechas se dan entre

- la práctica política y el mundo de la ciencia y la tecnología,

- las disciplinas científicas,

- los actores y gestores políticos y los ciudadanos,

- los expertos y los legos, y conducen a una nueva aproximación sistémica pero que comprende dos nuevos conceptos: uno de carácter estructural (espacio) y otro de dimensión funcional (gobernanza).

Dentro de esta visión sistémica, orientada preferentemente a comprender el modo en que las organizaciones generan innovaciones, la actividad científica ha tenido que combinar sus formas habituales de gobierno en las que sus actores, según se ha visto, ejercian funciones polivalentes como actores, gestores, políticos y usuarios, con otros modos encaminados a aumentar el número de funciones para conseguir una mayor conexión con la sociedad por medio de la asunción de crecientes cotas de responsabilidad y de intervención en los procesos de difusión y divulgación del conocimiento. El giro en el modo de producción de avances científicos y técnicos, cada vez más conectado con el mundo empresarial, ha colocado a los expertos "tecnocientíficos" ante un dilema: por un lado, ha aumentado su valor y participación en el área de la asesoría relacionada con la empresa: licencias, patentes, interacción a distintos niveles en empresas ("spin-offs", "start-ups"), pero, por otro lado, se ha incrementado la desconfianza hacia su independencia por parte de los sectores de la ciudadanía más críticos sobre la avenida del progreso y sus consecuencias.

En un orden parecido de cosas, se sitúa la intervención de la comunidad experta en problemas científico-tecnológicos de carácter público. Inmersos en una sociedad del riesgo, las actitudes del público ante nuevos productos o procesos tecnológicos son muy variadas y complejas, a cuyos retos de regulación y valoración deben tratar de enfrentarse los expertos, bien a través de agencias especificas o a título individual para ejercer el asesoramiento en instituciones de naturaleza generalista. En todos estos temas, la comunidad experta debe afrontar la búsqueda de confianza, junto a la necesidad de aportar conocimiento cualificado para una gestión estratégica de problemas de base científico-técnica y con incidencia en la agenda política como la seguridad, la política de $I+D$ en diferentes niveles, la introducción de nuevas tecnologias o la repercusión socioeconómica. 
NOTAS

1 Es particularmente relevante, en nuestra opinión, para darse cuenta de la fuerza con que irrumpe el concepto en la agenda política, a más alto nivel, analizar las conclusiones de la reunión del G8 celebrada en Alemania (Colonia) en 1999 (véase a este respecto, Muñoz, E., Santesmases, M.J., López Facal, J., Plaza, L.M. \& Todt, 0., 2005).

2 Véase, por ejemplo, el trabajo de François Ewald (1996) en el que se pone de manifiesto que el liberalismo no es la política que limitaria las obligaciones sociales a las obligaciones que caerían bajo la responsabilidad juridica, sino sobre la base de las relaciones entre derecho y moral, y de la identificación de los limites entre ambos tipos de obligaciones sociales.

3 La referencia a gestores es consecuencia de la visión orientada al rendimiento de lo público, pero puede extenderse a la utilización del término actores en sentido amplio.

4 Esta dificultad se manifiesta, por ejemplo, en R. A. Dahl (1947), reproducido en J.M Shafitz y A.C. Hyde, (1987). Observaciones sobre esta cuestión están en J.O. Wilson, (1989).

5 El concepto de estrategia aplicado al sector público surge en discusiones dentro de la institución. Para más detalles, véase $M$. H. Moore, obra citada, pág. 347 (notas a la págs. 71-72).

6 Una discusión general sobre el papel de la prensa en la función política está en Gary Orren, "Thinking about the Press and Government", citado en Moore (1995).

7 La evolución de los grupos de interés en la arena politica puede verse en las obras citadas.

8 Mancur Olson ha establecido una teoria económica para explicar las condiciones que permiten la formación de grupos de interés. Wilson (1973) presenta una teoria que sugiere un amplio conjunto de posibilidades para la formación de grupos de interés, incluyendo la idea de que los grupos pueden organizarse alrededor de «incentivos determinados dirigidos».
9 Este punto ha sido suscitado por Javier Echeverría, quien al revisar las primeras versiones de este texto, señaló certeramente que en el caso de la ciencia y la tecnología, la comunidad "tecnocientífican juega un papel polivalente, muy distinto al de los usuarios y consumidores en cualquier otro ámbito. Echeverria propone cualificar a ese colectivo "experto" como el "quinto poder" en la gobernanza de la ciencia y la tecnologia (véase J. Echeverría, 2003; y también J. Echeverria, 2002, con especial referencia al Capítulo 2, "Valores y Teoría de la acciónn).

10 Obra citada "El Siglo de la Ciencia», págs. 15-17, con referencia a Eric Hobsbawn.

\section{BIBLIOGRAFÍA}

Dahl, R. A. (1947): The Science of Public Administration, Public Administration Review 7, n¹ (Winter, 1947)

Echeverria, J. (2002): Ciencia y valores. Madrid: Ediciones Destino (Colección imago mundi vol. 7).

Echeverria, J. (2003): La revolución tecnocientifica. Madrid: Fondo de Cultura Económica de España.

Edler, J., Kuhlmann, S. \& Behrens, M. (eds) (2004): Changing Governance of Research and Technology Policy. The European Research Area. Cheltenham, UK., Northampton, MA, USA: Edward Elgar.

Ewald, F. (1996): Histoire de l' Etat Providence. Le Livre de Poche: Editions Grasset \& Fasquelle, 11ª edición.

Metcalfe, J. (1995): The economic foundations of technology policy: equilibrium and evolutionary perspectives, en: P. Stoneman (ed.), Handbook of Economics of Innovation and Technology Change. Oxford: Blackwell.

Moore, M.H. (1995): Creating public value. Strategic management in government. Cambridge, (Massachusetts), London (England): Harvard University Press.

Muñoz, E. (2002): New socio-political environments and the dynamics of European public research systems. Documento de Trabajo CTS 02-20. http://www.iesam.csic.es/doctrab.htm.

Muñoz, E., Santesmases, M.J., López Facal, J., Plaza, L.M. \& Todt, 0. (2005): El espacio común de conocimiento de la Unión Europea. Un enfoque al problema desde España. Documento de Trabajo, Madrid: Academia Europea de Ciencias y Artes España.

Olson, M. (1965): The Logic of Collective Action: Public Goods and the Theory of Groups. Cambridge, Mass: Harvard University Press.

Real Academia Española (2001): Diccionario de la lengua española. Madrid: RAE.

Sánchez Ron, J. M. (2000): El Siglo de la Ciencia. Madrid: Taurus Pensamiento, Grupo Santillana de Ediciones.

Seco, M., Andrés, O. \& Ramos, G. (1999): Diccionario del español actual. Madrid: Aguilar. 
Shafitz, J.M. \& A.C. Hyde, (eds.) (1987):

Classics of Public Administration, 2nd ed. Chicago: Dorsey Press.

Smits, R. \& Kuhlmann, S. (2004): The rise of systemic instruments in innovation, Int. J. Foresight and Innovation Policy. Vol 1, Nos 1-2, 4-32.

Truman, D.B. (1951): The Governmental Process: Political Interests and Public Opinion. New York: Knopf.

Websters Third International Dictionary (1986): Chicago, Auckland, Geneva, London, Manila, Paris, Rome, Seoul, Sydney, Tokyo, Toronto: Encyclopaedia Britannica Inc..

Wilson, J.Q. (1973): Political Organizations. New York: Basic Books.

Wilson, J.Q. (1989): Bureaucracy: What Government Agencies Do and Why They Do It. New York: Basic Books. 